\title{
Obesidade e COVID-19: Presente e Futuro
}

\section{Obesity and COVID-19: Present and Future}

Maria João GREGÓRIO $\rrbracket^{1,2}$, Alejandro SANTOS ${ }^{2}$, Pedro GRAÇA ${ }^{1,2}$

Acta Med Port 2021 May;34(5):329-331 - https://doi.org/10.20344/amp.15921

Palavras-chave: COVID-19; Obesidade

Keywords: COVID-19; Obesity

Com a chegada da COVID-19 à Europa e depois aos Estados Unidos, começou a avolumar-se a evidência que apontava a obesidade como uma das principais comorbilidades associadas a doença grave por COVID-19.

Um dos primeiros trabalhos sobre esta relação, que analisou as características e os outcomes clínicos de 383 doentes com COVID-19, na China, sugeria que os indivíduos com obesidade, em particular os do sexo masculino, tinham maior risco de desenvolver pneumonia grave. ${ }^{1}$ Estes primeiros indícios viriam a confirmar-se e atualmente a evidência sobre esta relação já é muito consistente. Uma revisão sistemática com meta-análise, que analisou um total de 75 estudos e 399461 participantes de 10 países, mostrou que a obesidade aumenta o risco de resultado COVID-19 positivo em mais de $46 \%$, de hospitalização em $113 \%$, de internamento em serviços de Medicina Intensiva em $74 \%$ e de mortalidade por COVID-19 em $48 \%{ }^{2}$

A COVID-19 foi declarada pela Organização Mundial da Saúde como pandemia há menos de um ano, contudo, a 6 de fevereiro de 2021 já é possível encontrar 1185 registos na PubMed quando pesquisam os termos 'obesity' e 'COVID-19'. O âmbito destes trabalhos é diverso, abrangendo, por exemplo, artigos que analisam a associação entre a obesidade e diferentes outcomes associados à COVID-19, e artigos que exploraram os possíveis mecanismos plausíveis para esta associação. Atualmente, a questão central reside na possibilidade da associação entre a obesidade e a COVID-19 ser de maior gravidade nos grupos mais jovens da população. Um estudo publicado recentemente na Circulation parece confirmar estas suspeitas, na medida em que mostra pela primeira vez que os efeitos adversos da obesidade em diferentes outcomes associados à COVID-19 são mais expressivos nos doentes com idade inferior a 50 anos, em comparação com os doentes com mais idade. ${ }^{3}$ Assim, no que diz respeito ao risco de COVID-19 grave, levanta-se a hipótese de que a 'idade metabólica' possa ser um fator tão importante quanto a idade cronológica.

Noutra revisão sistemática com meta-análise, que analisou um total 28 estudos e 6720 indivíduos, $41 \%$ des- tes apresentavam diversas comorbilidades. Este estudo mostrou que a probabilidade de ter um quadro grave de COVID-19 era muito superior nos doentes com história de doença cerebrovascular, cardiovascular, doença pulmonar crónica, cancro, diabetes e hipertensão. ${ }^{4}$ Tendo em conta a elevada prevalência destas patologias em Portugal e a sua associação com a obesidade, identificam-se aqui motivos para preocupação.

Estes dados são particularmente relevantes se considerarmos que o perfil de doentes hospitalizados em Portugal, em enfermaria mas também em serviços de Medicina Intensiva, tem vindo a alterar-se ao longo das diferentes vagas da pandemia. $O$ início da pandemia ficou marcado com um perfil de doentes mais velhos, mas progressivamente foi aumentando a proporção de doentes mais jovens a necessitar de hospitalização no decurso da infeção por SARS-CoV-2.

Estes resultados não são assim tão surpreendentes se se tiver em consideração a evidência gerada durante as pandemias anteriores, desde a pandemia da 'gripe espanhola' de 1918 aos surtos de 'gripe asiática' de 1957 e 1960, à 'gripe de Hong-Kong' de 1968 e até à pandemia de 2009 causada pelo vírus Influenza H1N1. A evidência gerada por estas pandemias já indiciava a relação entre a obesidade e as doenças infeciosas. ${ }^{5}$ Talvez a diferença relativamente ao momento atual reflita apenas a dimensão do problema da obesidade. Entre 1975 e os dias de hoje, a prevalência da obesidade na população, em geral, triplicou. ${ }^{6}$

Em Portugal, estudos já publicados analisaram as características e outcomes clínicos dos doentes com COVID-19 na população portuguesa sem incluírem a obesidade, pelo que ainda não é conhecido o seu impacto no número de hospitalizações, quer em enfermaria, quer em Cuidados Intensivos. A ausência da obesidade neste estudo foi recentemente destacada por Barbosa et al. ${ }^{7}$ De facto, a obesidade é ainda, por vezes, clinicamente negligenciada enquanto comorbilidade com efeito no prognóstico de algumas situações de doença.

Apesar disso, os relatos dos clínicos dos hospitais

1. Programa Nacional para a Promoção da Alimentação Saudável. Direção-Geral da Saúde. Lisboa. Portugal.

2. Faculdade de Ciências da Nutrição e Alimentação. Universidade do Porto. Porto. Portugal.

$\triangle$ Autor correspondente: Maria João Gregório. mariajoaobg@fcna.up.pt

Recebido: 09 de fevereiro de 2021 - Aceite: 10 de fevereiro de 2021 - First published: 20 de abril de 2021 - Online issue published: 03 de maio de 2021 Copyright $\odot$ Ordem dos Médicos 2021 
portugueses na linha da frente apontam para uma elevada prevalência de doentes hospitalizados com obesidade, e em particular para aqueles que se encontram em serviços de Medicina Intensiva. De igual modo, as orientações para a abordagem ao doente com suspeita/confirmação de COVID-19, bem como o estabelecimento de prioridades para a vacinação, têm acompanhado a evidência científica internacional sobre a identificação da obesidade como um fator de risco para COVID-19 grave. Estes dados levaram a que em Portugal a obesidade tenha sido associada à evolução para COVID-19 grave na Norma 004/2020 'COVID-19: Abordagem do Doente com Suspeita ou Confirmação de COVID-19', ${ }^{8}$ bem como na segunda fase do plano português de vacinação para a COVID-19, onde está incluído o grupo das pessoas com obesidade. Os ensaios das vacinas para a COVID-19 da Pfizer-BioNTech e da Moderna, apesar de limitados por um poder inadequado para comparar subgrupos e por uma incompleta estratificação de grupos de alto risco, parecem apresentar eficácia semelhante em indivíduos com e sem obesidade. Este dado é relevante, pois temia-se menor eficácia da vacinação nos doentes obesos face à resposta observada a outras vacinas. ${ }^{9}$

No contexto nacional, não só a obesidade é considerada nos documentos técnico-normativos que orientam a resposta concreta à COVID-19, mas também as políticas públicas de saúde em curso, como é o caso do Programa Nacional para a Promoção da Alimentação Saudável da Direção-Geral da Saúde, se organizaram no sentido de definir estratégias de ação para o contexto atual. The national food and strategy for the Portuguese COVID-19 response, publicada recentemente no European Journal of Clinical Nutrition, apresenta um modelo de intervenção a três níveis, no qual se destaca a importância da otimização do estado nutricional e do bom controlo metabólico de alguns grupos de risco para a COVID-19, nomeadamente nos indivíduos com pré-obesidade ou obesidade, propondo abordagens de intervenção concretas para o efeito. ${ }^{10}$ Destas destaca-se o modelo de aconselhamento breve para a alimentação saudável.

Em Portugal, a pré-obesidade e a obesidade afetam mais de 6,1 milhões de portugueses adultos, doença que tende a ser mais frequente nos grupos da população mais vulnerável (os mais pobres e com menos escolaridade). A obesidade e outras doenças crónicas, como a diabetes, hipertensão e doenças cardiovasculares são patologias onde o descontrolo metabólico se pode agravar em resultado da redução do acesso a cuidados de saúde e da crise económica e social que se vive atualmente. Esta situação, e em particular o crescimento das desigualdades em saúde nesta área, reforça a necessidade de se continuar a priorizar a implementação de políticas públicas intersectoriais para a prevenção e para o combate à obesidade.

São muitos os desafios sem precedentes na promoção da alimentação saudável durante a pandemia COVID e no pós-COVID. A par das fragilidades que já se identificaram no estado de saúde de muitos portugueses com obesidade e outras doenças crónicas associadas a hábitos alimentares inadequados, perspetivam-se outros desafios que podem condicionar a implementação das necessárias medidas para a modificação dos ambientes obesogénicos. Tanto a fragilidade da nossa economia (que abrange a proteção dos operadores económicos do setor alimentar), como a multiplicação dos discursos contra a implementação de medidas sanitárias mais restritivas, serão certamente ameaças à intervenção pública no combate à obesidade. Por último, valerá a pena destacar que o desafio de mudar estilos de vida (alimentação e atividade física) será ainda maior neste atual contexto de ansiedade, incerteza e exposição contínua a restrições severas, fruto das medidas que procuram controlar a propagação da COVID-19.

Alguns destes desafios, que se colocam atualmente às políticas de prevenção e controlo da obesidade na era COVID e pós-COVID, já começam a ser refletidos a nível internacional. Destaca-se o artigo 'Obesity and COVID-19: Blame isn't a strategy', a propósito da nova estratégia inglesa para a obesidade. Uma estratégia ambiciosa no que toca à tentativa de uma maior regulação do mercado dos alimentos poucos saudáveis, mas que acaba por ficar marcada pelas críticas à excessiva culpabilização e responsabilização individual que ficou patente no título do comunicado de imprensa da sua divulgação - 'Lose weight to beat COVID-19 and protect the NHS' - uma abordagem que os autores consideram ser típica da era COVID. ${ }^{11}$

Este será, pois, um período que exigirá um forte investimento na melhoria de prestação de cuidados nutricionais à população, facilitando o acesso a cuidados de saúde especializados e criando condições e modelos de prestação de cuidados que permitam otimizar a intervenção alimentar e nutricional.

\section{CONFLITOS DE INTERESSE}

Os autores declaram não ter conflitos de interesse relacionados com o presente trabalho.

\section{REFERÊNCIAS}

1. Cai Q, Chen F, Wang T, Luo F, Liu X, Wu Q, et al. Obesity and COVID-19 severity in a designated hospital in Shenzhen, China. Diabetes Care. 2020;43:1392-8.

2. Popkin B, Du S, Green W, Beck M, Algaith T, Herbst CH, et al. Individuals with obesity and COVID-19: A global perspective on the epidemiology and biological relationships. Obes Rev. 2020;21:e13128.

3. Hendren N, Lemos J, Ayers C, Das S, Rao A, Carter S, et al. Association of body mass index and age with morbidity and mortality in patients hospitalized with COVID-19. Circulation. 2021;143:135-44.

4. Honardoost M, Janani L, Aghili R, Emami Z, Khamseh M. The association between presence of comorbidities and COVID-19 severity: a systematic review and meta-analysis. Cerebrovasc Dis. 2021 (in press). doi: 10.1159/000513288.

5. Barbosa M, Lopes S, Fernandes V, Monteiro AM. Obesity and COVID-19: a forgotten relationship. Acta Med Port. 2021;34:158-70.

6. Moser JA, Galindo-Fraga A, Ortiz-Hernández AA, Gu W, Hunsberger $\mathrm{S}$, Galán-Herrera JF, et al. Underweight, overweight, and obesity as 
independent risk factors for hospitalization in adults and children from influenza and other respiratory viruses. Influenza Other Respir Viruses. 2019;13:3-9.

7. World Health Organization. Obesity and owerweight: key facts. 2020. [consultado 2021 fev 04]. Disponível em: https://www.who.int/newsroom/fact-sheets/detail/obesity-and-overweight.

8. Direção-Geral da Saúde. Norma 004/2020 - COVID-19: Abordagem do doente com suspeita ou confirmação de COVID-19. Lisboa: DGS; 2020.

9. Townsend M, Kyle T, Stanford F. COVID-19 vaccination and obesity: optimism and challenges. Obesity. 2021 (in press). doi: 10.1002/ oby. 23131

10. Gregório M, Irving S, Teixeira D, Ferro G, Graça P, Freitas G. The national food and nutrition strategy for the portuguese COVID-19 response. Eur J Clinical Nutr. 2021 (in press). doi: 10.1038/s41430-02000818-w.

11. The Lancet Diabetes Endocrinology. Obesity and COVID-19: blame isn't a strategy. Lancet Diabetes Endocrinol. 2020;8:731. 